\title{
Allergic diseases in children with attention deficit hyperactivity disorder: a systematic review and meta-analysis
}

Celine Miyazaki ${ }^{1}$, Momoko Koyama ${ }^{2}$, Erika Ota ${ }^{1,3^{*}}$, Toshiyuki Swa ${ }^{1,4}$, Linda B. Mlunde ${ }^{2}$, Rachel M. Amiya ${ }^{5}$, Yoshiyuki Tachibana ${ }^{6}$, Kiwako Yamamoto-Hanada ${ }^{7}$ and Rintaro Mori ${ }^{1}$

\begin{abstract}
Background: Reports of frequent manifestation of allergic diseases in children with attention deficit hyperactivity disorder (ADHD) have been the subject of mounting clinical interest. However, evidence supporting the association between ADHD and allergies is inconsistent and has yet to be systematically reviewed. The objective of this study was to compile and assess available studies on the association between ADHD and allergic diseases in children.

Methods: A comprehensive search using MEDLINE, EMBASE, the Cochrane library, and CINAHL databases was completed in 23 November 2015. The inclusion criteria for studies were that the research assessed allergic diseases in children, 18 years of age and younger, with a diagnosis of ADHD and that a distinct comparison group was incorporated. Any comparative studies, encompassing both randomized controlled trials and observational studies, were considered for inclusion. Two review authors independently assessed the quality of the selected studies by the use of validated assessment tools, performed data extraction and conducted meta-analysis according to Cochrane Collaboration guidelines.
\end{abstract}

Results: Five eligible studies were included in this systematic review. Of these studies, three were case-control and two were cross sectional studies. A majority of information from the five studies was classified as having low or unclear risk of bias. The meta-analysis showed an association between children with ADHD and asthma compared with the control groups (OR: 1.80, 95\% Cl: 1.57 - 2.07; five studies, low quality of evidence), but did not indicate an association between food allergy and ADHD (OR: 1.13, 95\% Cl: 0.88 - 1.47; three studies very low quality of evidence). The odds of experiencing allergic rhinitis, atopic dermatitis, and allergic conjunctivitis were slightly higher in children with ADHD compared with control groups, though a substantial statistical heterogeneity was notable in the overall effect estimates.

Conclusions: The findings from this review and meta-analysis show that children with ADHD are more likely to have asthma, allergic rhinitis, atopic dermatitis, and allergic conjunctivitis than their counterparts. Interventions including strategies for managing allergies in children with ADHD would be beneficial.

Keywords: Allergic conjunctivitis, Allergic disease, Allergic rhinitis, Asthma, Atopic dermatitis, Attention deficit hyper disorder, Coexisting condition, Food allergy, Meta-analysis

\footnotetext{
*Correspondence: ota@slcn.ac.jp

'Department of Health Policy, National Center for Child Health and

Development, 10-1-2 Okura, Setagaya-ku, Tokyo 157-8535, Japan

${ }^{3}$ Global Health Nursing, Graduate School of Nursing Science, St. Luke's

International University, 10-1 Akashicho, Chuo-ku, Tokyo 104-0044, Japan

Full list of author information is available at the end of the article
} 


\section{Background}

The association between attention deficit hyper disorder (ADHD) and allergic diseases, whether rooted in comorbidity or causality, has been a source of public and clinical concern since the 1980s [1]. The most recent estimate of worldwide ADHD prevalence in children and adolescents is reported at 7.1\% [2]. ADHD is often recognized in early-school children with persistent patterns of inattention and hyperactivity-impulsivity that interfere in functioning, social development or both and the symptoms usually persist to adulthood [3]. There are few therapy options (e.g., behavioral therapy or cognitive training) for children with ADHD, but the effectiveness varies depending on the individual because of the complex array of factors (e.g., genetic and environmental conditions) that intertwine with the behavioral interpretation during their development $[3,4]$. While a cure for ADHD has not yet been fully achieved, the available clinical treatment for children with ADHD, including adolescents and adults, is largely medication-based (e.g., methylphenidate, amphetamine or atomoxetine adrenergic agonistics drugs), and the prescribed medications are to improve certain aspects of attention span and hyperactivity behavior [5].

Whether ADHD is related to hypersensitivity or not has not been fully discerned, but numerous reports on allergies in children with ADHD have created a growing concern among healthcare providers. Several studies have documented cases of allergic manifestations (e.g., atopic dermatitis or asthma) in response to stimulant and non-stimulant drug treatments for ADHD, but the explanation for the allergic disease manifestations remained ambiguous [6-8]. In the aspect of the neurophysiological mechanism, some studies have suggested that the relationship between the immune response and the central nervous system (CNS) may predispose some children to autism, impulsive behavior or ADHD [9-14]. However, the proposed interrelated mechanism of the elevated proinflammatory cytokines reactivity in the brain found to be triggered by allergic response could does not adequately explain the different types of allergy manifested in the children with ADHD [15, 16]. With environmental factors (e.g., family or school setting and social distress) and other coexisting conditions (e.g., metabolic abnormalities, sleep disorders and epilepsy) being suggested to influence the severity of ADHD symptoms, it is necessary to gain a better understanding of the relationship between allergy and ADHD from both biological and epidemiological perspectives toward providing the most desirable care possible $[17,18]$.

Several epidemiological studies have reported that children with ADHD have a high risk of developing allergic diseases, such as asthma and atopic dermatitis, while other studies have reported no evidence of a link between allergy and ADHD [19-22]. Given such conflicting evidence on the association, it is suspected that a sizeable proportion of the ADHD population experiencing comorbidities with various allergic diseases may have been overlooked. Moreover, the resulting lack of clear recommendations in this area have meant that the optimal benefit of tailored interventions and supportive care may not reach those children in need. The objective of this systematic review was thus to compile and analyze the best available evidence on whether rates of allergic diseases are significantly higher in children with ADHD and to identify the specific types of allergic diseases to which such children may be prone.

\section{Methods}

\section{Search strategy}

This review was conducted in accordance with the Cochrane Handbook for Systematic Reviews and the Preferred Reporting Items for Systematic reviews and Meta-Analyses (PRISMA) statement, and the PRIMSA checklist is provided in an additional file (see Additional file 1) $[23,24]$. A search strategy was developed, with an information specialist, to meet the review question 'Is there an association between ADHD and allergies?' and the comprehensive search was completed on 23 November 2015 by the use of MEDLINE, EMBASE, the Cochrane library and CINAHL databases. To ensure the search was as comprehensive as possible, subject terms were exploded so as to include narrower terms, regardless their wide range word expressions in the text search. The search terms included 'child development disorders, pervasive,' 'mental disorders diagnosed in childhood,' 'attention deficit disorder,' 'learning disorder,' 'autism' and 'hypersensitivity' (see Additional file 2 for search strategy details). There was no date, language or types of publication restriction imposed on the search. The retrieved references and the articles were managed by the use of EndNote version X6 software (Copyright (c) 2012 Thomson Reuters).

\section{Study selection and quality assessment}

Five authors reviewed titles and abstracts of all potentially eligible articles retrieved from the databases. Three authors in one group and two authors in another group independently screened all the titles and abstracts from the bibliographic list retrieved, and the reference lists of the retrieved article were additionally hand-searched where necessary. After irrelevant studies were removed in this initial stage, two authors collected the full-text of the potentially relevant studies and independently examined the report content for determining eligibility based on our pre-specification criteria for including and excluding the studies. When studies referred to previously published protocols or results reported elsewhere, those 
referenced studies were retrieved and examined as well. After eligible studies had been selected, the studies were then coded by the first author's last name and publication year as their identification in this systematic review. Any disagreement was resolved by discussion with other authors or consultation with other experts in this subject.

The eligibility criteria for including studies for this review were developed by following the general methods for Cochrane reviews and the Non-randomised Studies Methods Group (NRSMG) of the Cochrane Collaboration guidelines [23]. The participants that met this review eligibility were children and adolescents who were 18 years of age or younger and were clinically diagnosed or identified through psychiatric assessment as having ADHD, and with the diagnosis of allergic diseases or immune hypersensitivity. Mixed-gender studies, singlegender studies and studies of ADHD-diagnosed children with or without record of receiving treatment were eligible for inclusion. The definition of ADHD was determined by the established Diagnostic and Statistical Manual of Mental Disorders, Fourth Edition, Text Revision (DSM-IV-TR) and DSM-5, and the National Institute for Health and Care Excellence (NICE) clinical guideline 72 on ADHD [25, 26]. In addition, studies that referenced use of structured diagnostic interviews and validated screening tools (e.g., Conners Parent Rating Scale (CPRS) or Child Behavior Checklist (CBCL) scales) in evaluating ADHD symptoms were considered eligible for inclusion. In regard to the diagnosis of allergic diseases, studies were considered for inclusion if the diagnosis was done under physical examination or laboratory test, or otherwise met the clinical criteria for the diagnosis of allergy (e.g., International Classification of Disease, Injuries, and Causes of Death [ICD]). There was no restriction placed on the types of study design included. Randomized controlled trials and all comparative studies (e.g., prospective, longitudinal, retrospective, case-control or cross sectional studies) with a comparison group of children without clinical indication of developmental disorders were all considered eligible for this review.

The exclusion criteria were studies that reported prespecified comorbidities within other categories of developmental disorders (e.g., autism spectrum disorders or specific learning disabilities) or neurological disorders, such as epilepsy. Finally, studies that included only adult population, studies without distinguishable comparison groups, studies using animal models, systematic reviews, case series reports, and articles that did not provide original data were considered irrelevant for inclusion.

To appraise the validity of the studies, two authors used risk of bias tools to perform quality and risk of bias assessment for all the eligible studies independently. The
Cochrane risk-of-bias assessment tool was used to assess clinical trial studies, and the Risk of Bias Assessment Tool for Non-randomized Studies (RoBANS), equivalent to that of the Cochrane risk-of-bias assessment tool, was used to assess the observational studies [27]. There were six main domains for potential bias evaluation in RoBANS and each domain was categorized as 'low risk', 'high risk', or 'unclear risk'. The methodological quality of the primary studies were examined and judged as either low, high or unclear risk of bias in relation to the assessment domains. To enhance consistency of judgment and decision making between the review authors, we additionally referred to the guidance from the Cochrane Risk of Bias Assessment Tool: for NonRandomized Studies of Interventions (ACROBAT-NRSI) that described the types of bias for selection of participants, confounding, measurement of interventions, measurement of outcomes, missing data and selection of reported results for clarification [28]. After the risk of bias assessment was completed, both authors independently extracted the data from the eligible primary studies and recorded the extracted data to a modified data collection form with items of required information listed. The information items were consistent with our prespecified criteria and were formatted to seek for the characteristic of the studies, setting, definition of the ADHD population, types of allergy and results of any outcome measures. The extracted data were then transferred to the Review Manager (RevMan) 5.3 software for meta-analysis [29].

\section{Data synthesis}

Studies with similar characteristic were combined for meta-analysis. When relevant outcome data were available for synthesis, they were entered into RevMan 5.3 software for pair-wise comparison. The Mantel-Haenszel method was used for assessing dichotomous outcomes, whereas for continuous outcomes, the inverse variance method would be used. The relative effect measures were calculated by using odds ratio (OR) statistics based on the reporting from the studies, and the relative effect estimate was assigned with a 95\% confidence interval (CI) and a $p$-value cut-off point of 0.05 . Since diagnosis tools used to assess ADHD were highly varied among the studies due to cultural and clinical practice differences across countries, the true effect would be most likely varied from one study to the next. For this reason, a random-effects model assumption was used. If the number of included studies was very small or the study designs were too diverse, both random-effects and fixedeffects models were used to test the trend of the estimated effect as well. To determine heterogeneity, the chi-squared method was used with a cut-off point of 0.10 to determine statistical significance. The $\mathrm{I}^{2}$ statistic 
was used to calculate consistency for the combined studies to test the impact of heterogeneity in the metaanalysis. When the reason for substantial heterogeneity was unclear, subgroup analysis was performed as a means of investigating heterogeneous results and identifying whether the difference between groups could have interaction to the effect magnitude. The sensitivity analysis was performed to examine whether the overall estimate in the meta-analysis was affected by studies that were different in their sampling approach (e.g., population-based designs as opposed to hospital, school, or community-based designs) or not.

\section{Grading of evidence}

To evaluate the quality of available data on the association between ADHD and allergy diseases, the Grading of Recommendations Assessment, Development and Evaluation (GRADE), supported by guidelines outlined in the GRADE handbook was used [30]. The strength of the evidence were divided into four quality grades: high, moderate, low, and very low, according to confidence in the estimate lying close to the true effect [31]. Determinations of quality were based on five factors: methodological limitations creating risk of bias within the study, inconsistency of results, indirectness of evidence, imprecision of results, and publication bias. Quality ratings for observational studies began from 'low', with the possibility of upgrading if further research would be likely to provide confidence in the estimate effect or no threats to validity and evidence of a dose-response or exposureresponse gradient were found. Studies were downgraded as 'very low' quality if they contained uncertainty about the directness of results or contained unsystematic observations [32].

\section{Results}

The comprehensive search identified a total of 261 studies from the databases (Fig. 1). After removing duplicates, 240 studies were screened by title and abstracts for potential inclusion. Out of 240 studies, 21 studies were selected for full-text examination. Of those 21 studies, 15 studies did not meet the entry criteria and were excluded with reasons provided (e.g., twin studies, studies with no specified comparison group or behavior assessments were made according to the allergic severity and not by ADHD clinical diagnoses; see Additional file 3 for details on excluded studies with reasons for exclusion). There were multiple reports of the same population identified and they were considered as one study for meta-analysis, but those multiple reports that showed to have overlapping results, only the study with the most relevant and high-quality data among the reports were selected for inclusion. There were no eligible RCT studies identified related to ADHD and allergies. After the final selection process, five studies, all observational in their study design, were found to meet the inclusion criteria. Of the five eligible studies, one was
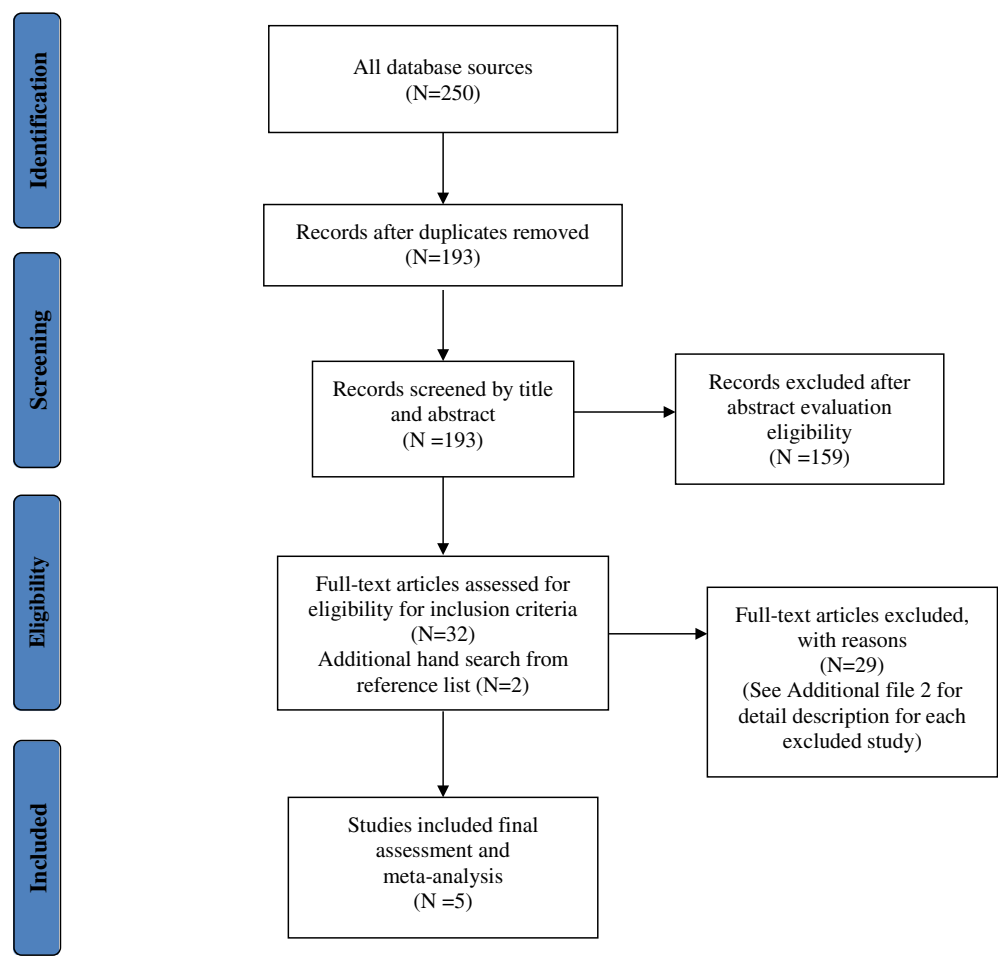

Fig. 1 Flow diagram for search process and selection of studies 


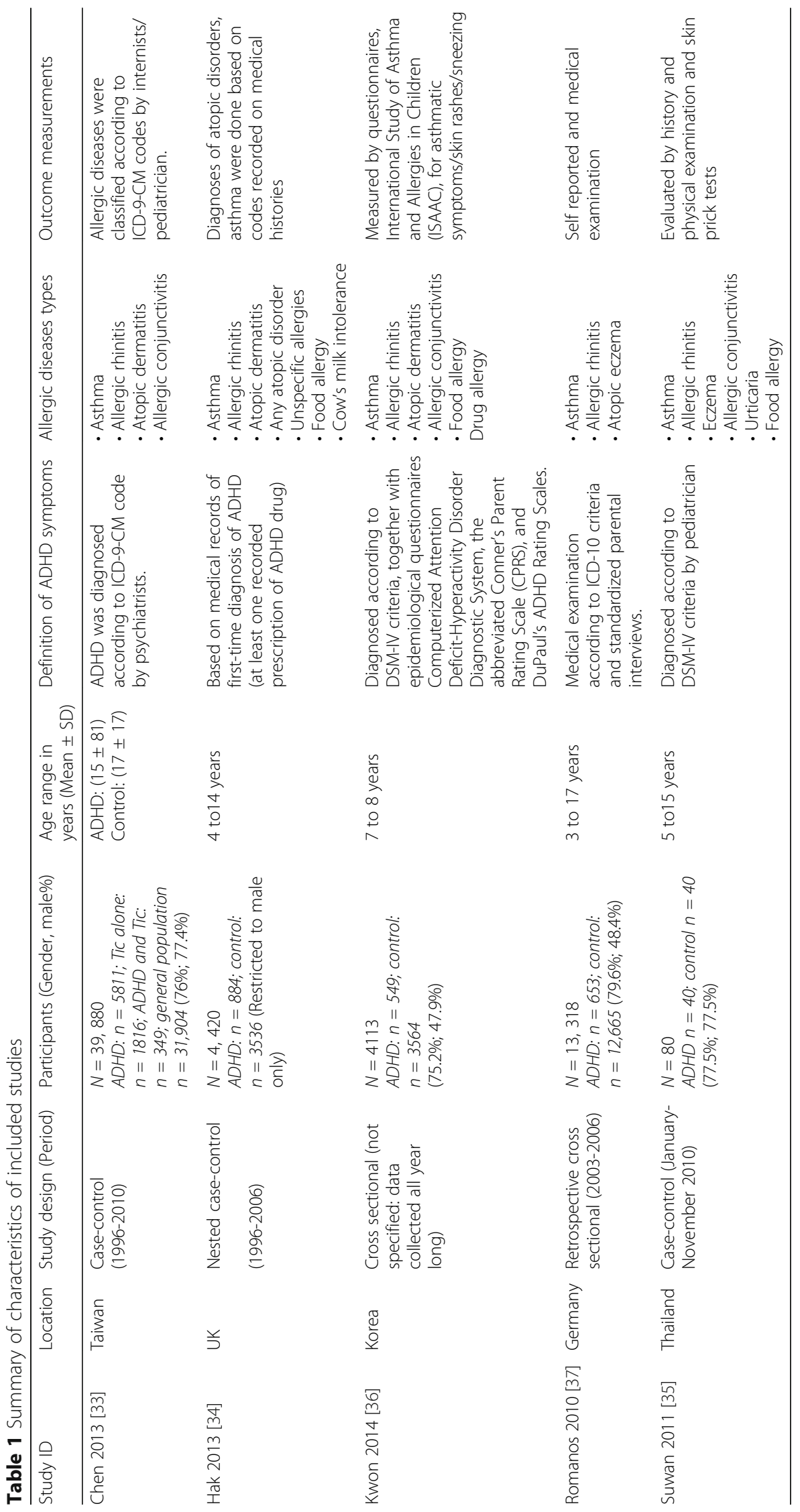


a population-based case-control study [33], two were case-control studies $[34,35]$, and two were cross sectional studies [36, 37] (Table 1). These studies were conducted in Germany, Korea, the United Kingdom (UK), Taiwan, and Thailand with varied data sources. Among these studies, one study [35] collected data from hospital patients and one study [36] collected data from the elementary schools. The remaining three studies collected data from national medical research databases: one study [33] used National Health Insurance Research Database (NHIR), one study [34] used UK General Practice Research Database (GPRD) and one study [37] used German Health Interview and Examination Survey for Children and Adolescents. Among the five included studies, only one study [34] indicated that children with ADHD had at least one record of a methylphenidate prescription within 12 moths after the date of first diagnosis. The rest of the four studies did not include background information regarding prescribed medications.

Of the five included studies, four studies [33-35, 37] were judged to have an unclear risk of bias and only one study [36] was judged to have a high risk of bias for selective outcome reporting based on RoBANS criteria (see Additional file 4 for the risk of bias assessment). With regard to the selection of participants, four studies were at unclear risk of bias [33, 34, 36, 37] whereas one study was at low risk of bias [35]. As for potential bias due to confounding, all studies were judged to have a low risk of bias. Regarding to measurement of exposure, four studies were at low risk of bias [33-35, 37] and one study was at unclear risk of bias [36]. In the blinding of outcome assessment domain, one study was at low risk of bias [37] but the rest of the four studies were at unclear risk of bias. All the studies were at low risk of bias in terms of bias caused by incomplete of outcome data. As for selective outcome reporting, two studies were at unclear risk of bias [33,34], one study was at high risk of bias [36], and two studies were at low risk of bias [35, 37]. Further details on the supporting judgments for risk of bias assessments are presented in Additional file 4.

There was a total of 61,811 children involved in the five included studies and 38,324 (62\%) of the children were males. Of the 61,811 children, 7937 (13\%) were diagnosed with ADHD. The types of allergic diseases reported in these studies were asthma, allergic rhinitis, atopic dermatitis, and allergic conjunctivitis. Other allergies such as food allergy, drug allergy, urticatia, any atopic disorder or unspecified allergies were also reported in some studies (see Additional file 5 for a summary of results on allergic diseases in children with ADHD). In performing the meta-analysis, studies were stratified by their sample selection approach as part of the sensitivity analysis. Two studies $[33,37]$ were of population-based design and they were grouped as (nationwide studies). As for the remaining three studies [34-36], the data were collected from selected schools or hospitals; wherefore, it was grouped as (institutional-based studies).

\section{Asthma}

Five studies examined the risk of asthma in children with ADHD compared to children without ADHD (Fig. 2a). The meta-analysis showed that children with ADHD were nearly twice as likely to have asthma compared with those in the control groups (OR: 1.80, 95\% CI: 1.57 $-2.07, \mathrm{I}^{2}=60 \%$; five studies, $n=59,646$ children). The pooled estimate from the studies showed a statistically significant difference between the two groups, and the large overall estimate indicated an association between asthma and ADHD. The heterogeneity across the studies was found to be statistically non-significant but a moderate inconsistency was detected. To explore the influence of nationwide studies versus institutional-based studies on the effect estimate, subgroup analysis was conducted. There was no significant difference found between the nationwide studies [33, 37] and the institutional-based studies [34-36].

\section{Allergic rhinitis}

Five studies evaluated the risk of allergic rhinitis in children with ADHD compared to those without ADHD (Fig. 2b). The result from the combined studies showed that a higher proportion of children in the ADHD groups had allergic rhinitis compared to the control groups (OR: 1.59, 95\% CI: $1.13-2.23, \mathrm{I}^{2}=93 \%$; five studies, $n=59,646$ children). This suggests that children with ADHD experienced 59\% greater odds of having allergic rhinitis relative to the children without ADHD; however, substantial evidence of heterogeneity and inconsistency were detected among the studies. Regarding to subgroup analysis, there was no significant difference indicated between the nationwide studies [33, 37] and the institutional-based studies [34-36].

\section{Atopic dermatitis}

Five studies assessed the association between atopic dermatitis and ADHD in children (Fig. 2c). From the pooled estimated across the studies, the relative effect indicated a significant difference between the ADHD and the control groups, with cosiderable heterogeneity and inconsistency found across the studies (OR: 1.43, 95\% CI: $1.09-1.88, \mathrm{I}^{2}=87 \%$, five studies, $\left.n=59,646\right)$. In the subgroup analysis, an apparent difference was detected $(p=0.006)$. The nationwide studies $[33,37]$ showed that the children in the ADHD groups had higher odds of atopic dermatitis relative to the control groups (OR: $1.74,95 \%$ CI: $1.36-2.22, \mathrm{I}^{2}=79 \%$; two studies, $n=51,033$ children), and there was a substantial heterogeneity and inconsistency found between the 


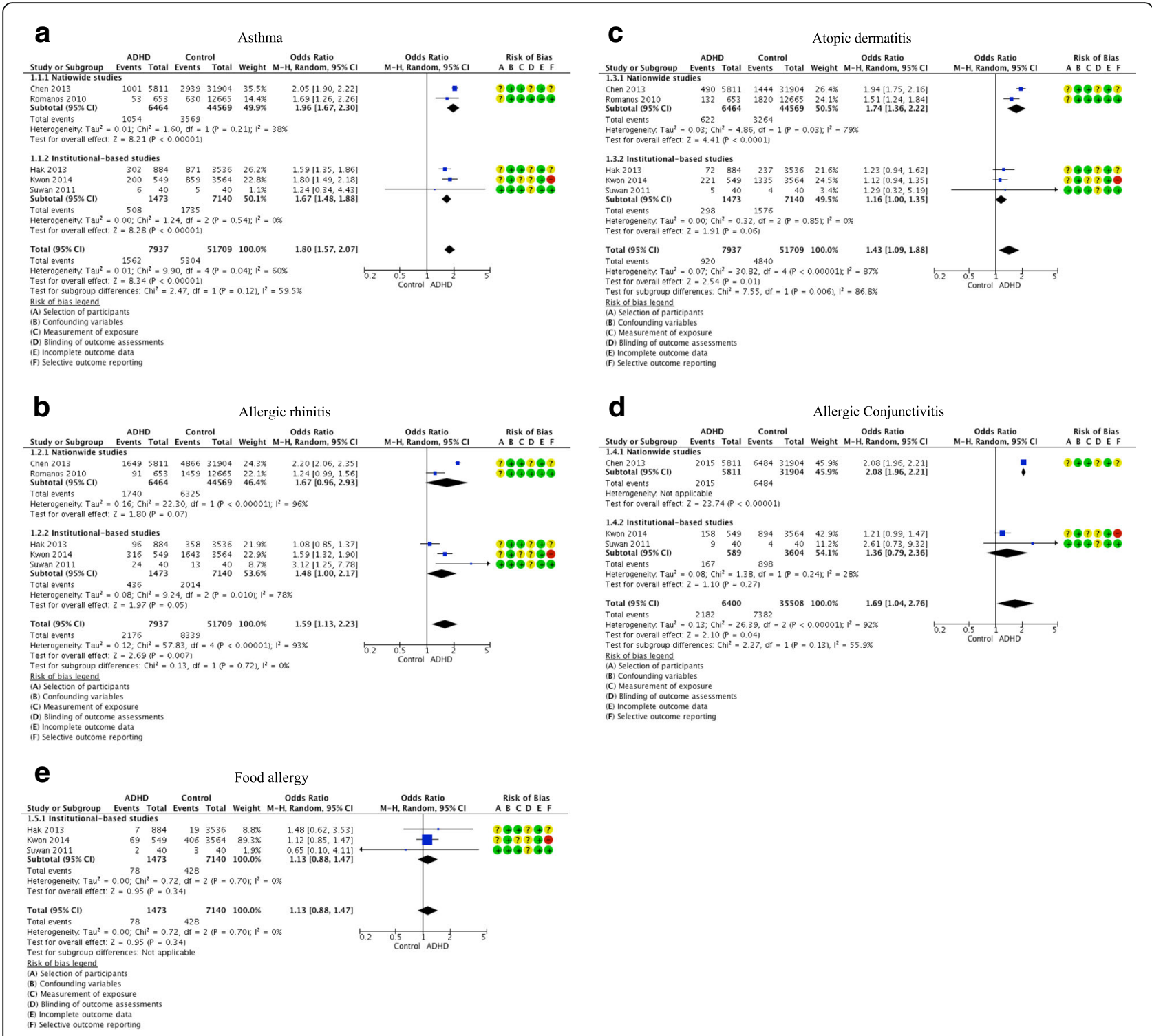

Fig. 2 Forest plots of allergic diseases in children with ADHD and in control children. a Asthma. b Allergic. c Atopic dermatitis. d Allergic conjunctivitis. e Food allergy.

studies. From the institutional-based studies [34-36], the odds of atopic dermatitis appeared to be only slightly higher in the ADHD groups compared with the control groups (OR: $1.16,95 \% \mathrm{CI}: 1.00-1.35, \mathrm{I}^{2}=0 \%$; three studies, $n=8613$ children), and the effect estimate did not reach statistical significance as the confidence interval crossed the line of null effect.

\section{Allergic conjunctivitis}

Three studies provided data for allergic conjunctivitis in children with ADHD compared with children without ADHD (Fig. 2d). The pooled estimate from these studies showed that a higher proportion of children in the ADHD groups experienced allergic conjunctivitis compared with the control groups (OR: 1.69, 95\% CI: 1.04 - 2.76; $\mathrm{I}^{2}=92 \%$, three studies, $\left.n=41,908\right)$; however, there was notable heterogeneity and a substantial inconsistency detected across the studies. Differences between the subgroups, meanwhile were not statistically significant.

\section{Food allergy}

Three studies examined the association between ADHD and food allergy; all of which were institutional-based studies (Fig. 2e). The meta-analysis showed no significant difference between the ADHD groups and the control groups, as the confidence interval of the odds ratio estimate included the the null value (OR: 1.13, 95\% CI: $0.88-1.47, \mathrm{I}^{2}=0 \%$; three studies, $\left.n=8613\right)$. There was 
no implication of inconsistency and heterogeneity between the studies in the pooled result.

\section{Quality of evidence}

The quality of evidence for asthma associated with ADHD in children was considered low quality, largely due to information from one study [36] contributing a serious risk of bias, thus posing a major threats to the validity of information derived from the studies (Table 2). As for allergic rhinitis, atopic dermatitis, allergic conjunctivitis and food allergy, the quality of the evidence was downgraded from low to very low for serious risk of bias and inconsistency between the included studies. Details on the specific factors, such as overall risk of bias, inconsistency, indirectness, imprecision and publication bias, that influenced the evidence quality are described in the summary of evidence quality table, based on the GRADE approach (see Additional file 6). Publication bias assessment was considered to be inappropriate since only five studies were identified in this review. As a test for funnel plot symmetry should ideally include at least ten studies, the test for publication bias was not adequately powered to determine whether this was truly an issue in our analyses.

\section{Discussion}

This systematic review was designed to compile and present the best available evidence for the association between children with ADHD and allergic diseases in children - a relationship hitherto not yet addressed in a randomized controlled trial. Our meta-analysis of the identified observational studies indicated that children with ADHD have an $80 \%$ increased odds of asthma compared with children without ADHD. The high asthma rate in children with ADHD could potentially be linked to the recent discovery of genetic association, in which several studies suggested that a gene polymorphism of dopamine receptor D5 (DRD5), a form of the dopamine D1-like receptor, is associated with a ADHD behavior subtype and that the expression of DRD5 is found in both the mammalian brain and peripheral blood leukocytes [38-40]. According to these reports, DRD5 may also engage in some part of the immunological regulation process of $\mathrm{T}$ helper 17 cell (Th17) differentiation, which is extensively involved in asthma development [41]. Available literature thus seemingly supports the possible comorbidity of ADHD and asthma, but the correlation between these multiple mechanisms has not yet been clarified.

For allergic rhinitis, atopic dermatitis, and allergic conjunctivitis, the estimated relative odds were slightly higher in children with ADHD than in children without ADHD. The low statistical power most likely resulted from the statistical heterogeneity and the methodological diversity of the included studies, with a further potential confounder in factors that were not measured (e.g., climate, pollution or microbial agents). Since allergic rhinitis and conjunctivitis are triggered by seasonal or household allergens, insufficient covariate adjustment from the primary studies could yield heterogeneity in the meta-analyses; for example, cross sectional data may have been collected during a period when particular allergens are naturally more prevalent [42]. Although the overall odds ratio for atopic dermatitis was deemed small, the nationwide studies from the subgroup analysis showed a significantly higher rate of atopic dermatitis in children with ADHD than in the control groups, and interestingly, the estimate was similar to the result for asthma. In line with that observation, a recent study of atopic dermatitis reported that there was a high frequency of filaggrin $(F L G)$ null alleles detected in children of European origin with both atopic dermatitis and asthma phenotype [43, 44]. Perhaps it would be advantageous to more closely examine in future studies the comorbidity of atopic allergies and asthma in children with ADHD, particularly, whether the allergic march, in terms of the progression of various allergic conditions with age, and genetic penetrance could be a potential link.

Regarding to food allergy, there was no significant difference found between children with ADHD and children in the control groups. One underlying reason for this could be the complexity of the Immunoglobulin $\mathrm{E}$ (IgE) immune response to food allergens in the gastrointestinal tract falling between the tolerance and sensitization mechanisms [45]. In line with the present findings, a previous systematic review similarly found no evidence for an association between serum-IgE levels and ADHD symptoms [20]. Although the complex genetic associations between ADHD and immunological regulations in the CNS have been emphasized in this review, considering the onset and the vast array of neuropsychiatric disorder outcomes, neuronal signaling and immune regulation thus represented only a small part of the pathogenesis $[10,46]$.

The overall quality of evidence for an association between allergic diseases and ADHD children was found to be low for asthma and very low for atopic dermatitis, allergic rhinitis, allergic conjunctivitis, and food allergy. The differences of study designs and studies with risk of bias were largely attributed to the downgrading of the quality. Perhaps more case-control studies with standardized protocols could allow for better effect estimation and minimize the risk of bias posed in observational studies, thus improving the validity of meta-analyses in future.

This review provides a summary of the overall level of evidence for an association between ADHD and 
Table 2 Summary of findings

\begin{tabular}{|c|c|c|c|c|c|}
\hline \multicolumn{6}{|c|}{$\begin{array}{l}\text { Allergy diseases in children with ADHD } \\
\text { Population: Children with ADHD } \\
\text { Setting: Germany, Korea, Taiwan, Thailand, UK } \\
\text { Intervention: none (observation of the difference in risk of allergy diseases) } \\
\text { Comparison: Children without ADHD } \\
\text { Outcome: Allergic diseases }\end{array}$} \\
\hline \multirow[t]{2}{*}{ Allergy diseases } & \multicolumn{2}{|c|}{ Anticipated absolute effects* $(95 \% \mathrm{Cl})$} & \multirow{2}{*}{$\begin{array}{l}\text { Relative effect } \\
(95 \% \mathrm{Cl})\end{array}$} & \multirow{2}{*}{$\begin{array}{l}\text { № of participants } \\
\text { (studies) }\end{array}$} & \multirow{2}{*}{$\begin{array}{l}\text { Quality of the } \\
\text { evidence (GRADE) }\end{array}$} \\
\hline & $\begin{array}{l}\text { Control group risk } \\
\text { (Assumed risk) }\end{array}$ & $\begin{array}{l}\text { ADHD group risk } \\
\text { (Corresponding risk) }\end{array}$ & & & \\
\hline Asthma & 125 per 1000 & 205 per 1000 (183 to 228$)$ & OR 1.80 (1.57 to 2.07 ) & 59,646 (5 studies) & $\oplus \oplus O \circ$ LOW $^{a, h}$ \\
\hline a) Nationwide studies & a) 71 per 1000 & a) 130 per 1000 (113 to 149$)$ & a) OR 1.96 (1.67 to 2.30 ) & a) 51,033 (2 studies) & a) $\oplus O O O$ VERY LOW ${ }^{b, h}$ \\
\hline $\begin{array}{l}\text { b) Institutional-based } \\
\text { studies }\end{array}$ & b) 241 per 1000 & b) 347 per 1000 (320 to 374 ) & b) OR 1.67 (1.48 to 1.88 ) & b) 8613 (3 studies) & b) $\oplus O O O$ VERY LOW ch \\
\hline Allergic rhinitis & 153 per 1000 & 222 per 1000 (169 to 286$)$ & OR 1.59 (1.13 to 2.23$)$ & $\begin{array}{l}\text { 59,646 (5 studies) } \\
\text { a) }\end{array}$ & ๑OOO VERY LOW a,d,e,h \\
\hline a) Nationwide studies & a) 134 per 1000 & a) 205 per 1000 (129 to 312) & a) OR 1.67 (0.96 to 2.93) & b) 51,033 (2 studies) & a) $\oplus O O O$ VERY LOW b,d,e,h \\
\hline $\begin{array}{l}\text { b) Institutional-based } \\
\text { studies }\end{array}$ & b) 325 per 1000 & b) 416 per 1000 (325 to 511) & b) OR 1.48 (1.00 to 2.17 ) & c) 8613 (3 studies) & b) $\oplus 000$ VERY LOW ${ }^{c, d, e, h}$ \\
\hline Atopic dermatitis & 100 per 1000 & 137 per 1000 (108 to 173 ) & OR 1.43 (1.09 to 1.88$)$ & 59,646 (5 studies) & ๑OOO VERY LOW ${ }^{\mathrm{a}, \mathrm{d}, \mathrm{h}}$ \\
\hline a) Nationwide studies & a) 94 per 1000 & a) 154 per 1000 (124 to 188) & a) OR 1.74 (1.36 to 2.22 ) & a) 51,033 (2 studies) & a) $\oplus 000$ VERY LOW b,d,h \\
\hline $\begin{array}{l}\text { b) Institutional-based } \\
\text { studies }\end{array}$ & b) 100 per 1000 & b) 114 per 1000 (100 to 130$)$ & b) OR 1.16 (1.00 to 1.35$)$ & b) 8613 (3 studies) & b) $\oplus O O O$ VERY LOW ch \\
\hline Allergic Conjunctivitis & 203 per 1000 & 301 per 1000 (210 to 413 ) & OR 1.69 (1.04 to 2.76) & 41,908 (3 studies) & ๑O०O VERY LOW c,d,e,h \\
\hline a) Nationwide studies & a) 203 per 1000 & a) 347 per 1000 (333 to 360) & a) OR 2.08 (1.96 to 2.21 ) & a) 37,715 (1 study) & a) $\oplus \oplus \circ O$ LOW $^{f}$ \\
\hline $\begin{array}{l}\text { b) Institutional-based } \\
\text { studies }\end{array}$ & b) 175 per 1000 & b) 224 per 1000 (144 to 334) & b) OR 1.36 (0.79 to 2.36 ) & b) 4193 (2 studies) & b) $\oplus O O O$ VERY LOW ${ }^{g, d, e, h}$ \\
\hline $\begin{array}{l}\text { Food allergy } \\
\text { Institutional-based } \\
\text { studies }\end{array}$ & 75 per 1000 & 84 per 1000 (67 to 106) & OR 1.13 (0.88 to 1.47 ) & 8613 (3 studies) & ๑OOO VERY LOW c,e,h \\
\hline \multicolumn{6}{|c|}{$\begin{array}{l}\text { *The risk in the ADHD group (and its } 95 \% \text { confidence interval) is based on the assumed risk in the comparison group and the relative effect of the exposure (and } \\
\text { its } 95 \% \text { Cl) } \\
\text { CI confidence interval, OR odds ratio } \\
\text { GRADE Working Group grades of evidence (level of evidence of grading for observational studies) } \\
\text { Very low: Observational studies with uncertainty about the directness of results or unsystematic observations } \\
\text { Low: Observational studies with no threats to validity } \\
\text { Moderate: Observational studies with no threats to validity and evidence of a dose-response or exposure-response gradient } \\
\text { High: Observational studies with no threats to validity yielding very large effects } \\
\text { a Four of the studies had limitation on the selection of participants and blinding of outcomes assessments by their study designs but one study had unclear risk } \\
\text { of bias in the measurement of exposure and one study indicated high risk of bias on the outcome reporting which lowered the quality of the } \\
\text { observational evidence } \\
\text { b The proportion of information was from two studies indicated with limitation on selection of participants and blinding by their study designs but the unclear } \\
\text { risk of selective reporting which lowered the quality of the observational evidence } \\
\text { ' The proportion of information was from two studies indicated limitation on selection of participants and blinding of outcome assessments by their study } \\
\text { designs but one study had high risk of outcome reporting, which lowered the quality of the observational evidence } \\
\text { d There is an indication of significant inconsistency }\left(I^{2}>80 \%\right) \\
\text { e Information were from high heterogeneity and small sample size with a wide confidence interval } \\
\text { f The information is based on one study, which had limitation on the selection of participants, blinding of outcomes assessments and selective outcome reporting } \\
\text { by the study design } \\
9 \text { The information from two studies that had limitation on selection of participants, measurement of exposure, blinding of outcome assessment by their study } \\
\text { designs but one with high risk of bias on selective outcome reporting which lowered the quality of the observational evidence } \\
\text { h The possibility of publication bias is not disregarded but it was not considered to downgrade the quality of the observational evidence }\end{array}$} \\
\hline
\end{tabular}

allergies. However, certain limitations should be considered when interpreting this review. The evidence from these studies pertains only to the association between ADHD and allergies under the speculation of possibly coexisting conditions and not on the onset and the causative pathways between the two conditions. Furthermore, this review was not able to obtain information on the stimulant or non-stimulant medication used in the ADHD population, except for in one study [34], where the author mentioned that the children with ADHD had at least one prescription record of methylphenidate. Since that study excluded those who did not used methylphenidate from the assessment, a comparison group was not available for analysis, and the evidence of an association between stimulant used for ADHD and allergies remains 
lacking. In several of the identified studies [33, 34, 37], the control group of children was selected based on the absence of ADHD medical records with one study excluded female children; therefore, potential ADHD cases in the control group were not considered. With a small sample size in one study, an overestimation of power for the pooled standardized mean could likely influence the magnitude of the observed relationships between ADHD and allergic diseases. To enhance the quality of evidence, the presence of potential confounding factors in terms of cognitive ability, family environment (e.g., parents' mental health, parenting attitudes), and other potential coexisting mental diseases (e.g., anxiety disorder, major depressive disorder, autism spectrum disorder) should be elucidated. Additionally, medication sensitivity, bacterial or fungal infection and preterm birth should also be adequately identified and adjusted for in analyses.

\section{Conclusions}

This systematic review showed that children with ADHD had elevated rates of asthma compared to the children without ADHD. An association between food allergy and ADHD, meanwhile, was not evident based on the meta-analysis. The odds of allergic rhinitis, atopic dermatitis, and allergic conjunctivitis in children with ADHD was found to be slightly higher than in children without ADHD, though the overall effect size was affected by substantial heterogeneity across the studies. Interventions incorporating strategies that focus on allergic disease management and collaborative care for children with ADHD might be beneficial to explore.

\section{Additional files}

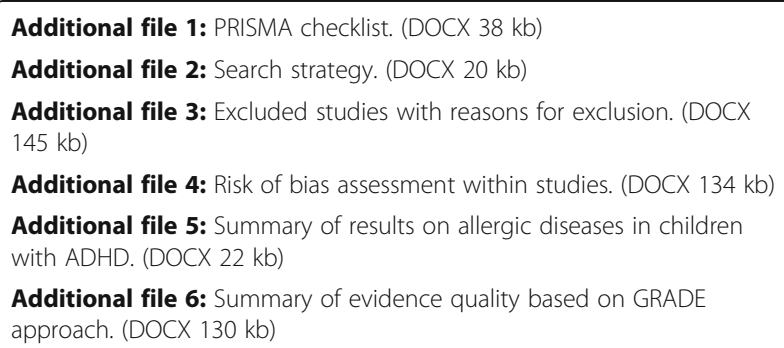

\section{Abbreviations}

ACROBAT-NRSI: Cochrane risk of bias assessment tool: for non-randomized studies of Interventions; ADHD: Attention deficit hyperactivity disorder; CBCL: Child behavior checklist; Cl: Confidence interval; CNS: Central nervous system; CPRS: Conners parent rating scale; DAT: Dopamine transporter; DMS-IV-TR: Diagnostic and statistical manual of mental disorders, fourth edition, text revision; DRD5: Dopamine receptor D5; DSM-5: Diagnostic and statistical manual of mental disorders, fifth edition; FLG: Gene encoding filaggrin; GRADE: The grading of recommendations assessment, development and evaluation; ICD: The international classification of diseases, injuries, and causes of death; IgE: Immunoglobulin E; ISAAC: International study of asthma and allergies in children; NICE: National Institute for Health and Care Excellence; NRSMG: Non-randomised Studies Methods Group; OR: Odds ratio; PRISMA: Preferred reporting items for systematic reviews and meta-analyses; RoBANS: Risk of bias assessment tool for non-randomized studies; Th17: T helper 17 cell

\section{Acknowledgments}

We would like to acknowledge the help of Ms. Chiemi Kataoka, Ms. Miwako Segawa and Ms. Yuko Serizawa at the National Research Institute for Child Health and Development for collecting studies and updating the database searches.

\section{Funding}

All phases of this study were supported by a National Center for Child Health and Development Grant (26A-5) and The Clinical Research Program for Child Health and Development, AMED (No. 27300101). None of these sources participated in any part of the performance of the study.

\section{Availability of data and materials}

All data and materials, on which the conclusions of the manuscript rely, are included in the main paper and presented in the additional supporting files.

\section{Authors' contributions}

CM contributed to the study concept and design, identification of eligible studies, study selection, data collection, data extraction, data analysis, data interpretation, and drafting and critical revision of the manuscript. MK participated in the identification of studies, data collection, data extraction, and data analysis. EO participated in the study concept and design, identification of studies, data interpretation, and critical revision of the manuscript. RMA and LBM participated in the identification of eligible studies at the initial screening stage and revision of the manuscript. TS coordinated the search strategy and conducted the database search for this review. YT, KY-H and RM contributed critical revision of the manuscript for important intellectual content. All authors read and approved the final manuscript for submission and agreed to be accountable for all aspects of the work.

\section{Competing interests}

The authors declare that they have no competing interests.

\section{Consent for publication}

Not applicable.

\section{Ethics approval and consent to participate}

This systematic review and meta-analysis was conducted in accordance with the World Medical Association's Declaration of Helsinki ethical principles revised in 2013 for medical research involving human subjects, including research on identifiable human material and data. This study did not report on or involve the use of any animal, individual person's data in any form, human data or tissue.

\section{Publisher's Note}

Springer Nature remains neutral with regard to jurisdictional claims in published maps and institutional affiliations.

\section{Author details}

${ }^{1}$ Department of Health Policy, National Center for Child Health and Development, 10-1-2 Okura, Setagaya-ku, Tokyo 157-8535, Japan. ${ }^{2}$ Department of Community and Global Health, Graduate School of Medicine, The University of Tokyo, 7-3-1 Hongo, Bunkyo, Tokyo 113-8654, Japan. ${ }^{3}$ Global Health Nursing, Graduate School of Nursing Science, St. Luke's International University, 10-1 Akashicho, Chuo-ku, Tokyo 104-0044, Japan. ${ }^{4}$ Graduate School of Human Sciences, Osaka University, 1-1 Yamadaoka Suita, Osaka Prefecture 565-0871, Japan. ${ }^{5}$ Department of Family Nursing, Graduate School of Medicine, The University of Tokyo, 7-3-1 Hongo, Bunkyo, Tokyo 113-8654, Japan. 'Department of Psychosocial Medicine, National Center for Child Health and Development, 10-1-2 Okura, Setagaya-ku, Tokyo 157-8535, Japan. ${ }^{7}$ Department of Medical Specialties, National Center for Child Health and Development, 10-1-2 Okura, Setagaya-ku, Tokyo 157-8535, Japan. 
Received: 3 June 2016 Accepted: 23 March 2017

Published online: 31 March 2017

\section{References}

1. Marshall P. Attention deficit disorder and allergy: a neurochemical model of the relation between the illnesses. Psychol Bull. 1989;106(3):434-46.

2. Thomas R, Sanders S, Doust J, Beller E, Glasziou P. Prevalence of attentiondeficit/hyperactivity disorder: a systematic review and meta-analysis. Pediatrics. 2015:135(4):e994-1001.

3. American Psychiatric Association. Diagnostic and statistical manual of mental disorders 5th ed. Washington: American Psychiatric Association Press; 2013.

4. August GJ, Realmuto GM, MacDonald III AW, Nugent SM, Crosby R. Prevalence of ADHD and comorbid disorders among elementary school children screened for disruptive behavior. J Abnorm Child Psychol. 1996; 24(5):571-95.

5. Voeller KK. Attention-deficit hyperactivity disorder (ADHD). J Child Neurol. 2004;19(10):798-814

6. Warshaw EM, Paller AS, Fowler JF, Zirwas MJ. Practical management of cutaneous reactions to the methylphenidate transdermal system: recommendations from a dermatology expert panel consensus meeting. Clin Ther. 2008:30(2):326-37.

7. Vashi NA, Souza A, Cohen N, Franklin B, Cohen DE. Allergic contact dermatitis caused by methylphenidate. Contact Dermatitis. 2011;65(3):183-5.

8. Wigal SB, McGough JJ, McCracken JT, Biederman J, Spencer TJ, Posner KL, Wigal TL, Kollins SH, Clark TM, Mays DA, et al. A laboratory school comparison of mixed amphetamine salts extended release (Adderall XR) and atomoxetine (Strattera) in school-aged children with attention deficit/ hyperactivity disorder. J Atten Disord. 2005;9(1):275-89.

9. Chang HY, Seo J-H, Kim HY, Kwon J-W, Kim B-J, Kim HB, Lee S-Y, Jang GC, Song DJ, Kim WK, et al. Allergic diseases in preschoolers are associated with psychological and behavioural problems. Allergy Asthma Immunol Res. 2013;5(5):315-21.

10. Comings DE, Gade-Andavolu R, Gonzalez N, Wu S, Muhleman D, Blake H, Chiu F, Wang E, Farwell K, Darakjy S, et al. Multivariate analysis of associations of 42 genes in ADHD, ODD and conduct disorder. Clin Genet. 2000;58(1):31-40.

11. Lee $\mathrm{YH}$, Song GG. Meta-analysis of case-control and family-based associations between the 5 -HTTLPR L/S polymorphism and susceptibility to ADHD. J Atten Disord. 2015

12. Merikangas KR, Calkins ME, Burstein M, He J-P, Chiavacci R, Lateef T, Ruparel K, Gur RC, Lehner T, Hakonarson H, et al. Comorbidity of physical and mental disorders in the neurodevelopmental genomics cohort study. Pediatrics. 2015;135(4):e927-38.

13. Petra Al, Panagiotidou S, Hatziagelaki E, Stewart JM, Conti P, Theoharides TC. Gut-microbiota-brain axis and its effect on neuropsychiatric disorders with suspected immune dysregulation. Clin Ther. 2015;37(5):984-95.

14. Miyazaki C, Koyama M, Ota E, Swa T, Amiya RM, Mlunde LB, Tachibana Y, Yamamoto-Hanada K, Mori R. Allergies in children with autism spectrum disorder: a systematic review and meta-analysis. Rev J Autism Dev Disord. 2015;2(4):374-401.

15. Hornig M. The role of microbes and autoimmunity in the pathogenesis of neuropsychiatric illness. Curr Opin Rheumatol. 2013;25(4):488-795.

16. Isgren A, Jakobsson J, Pålsson E, Ekman CJ, Johansson AG, Sellgren C, Blennow K, Zetterberg H, Landén M. Increased cerebrospinal fluid interleukin-8 in bipolar disorder patients associated with lithium and antipsychotic treatment. Brain Behav Immun. 2015;43:198-204.

17. Gillberg C, Gillberg IC, Rasmussen P, Kadesjö B, Söderström H, Råstam M, Johnson M, Rothenberger A, Niklasson L. Co-existing disorders in ADHD implications for diagnosis and intervention. Eur Child Adolesc Psychiatry. 2004;13(Suppl 1):i80-92.

18. Subcommittee on Attention-Deficit/Hyperactivity Disorder, Steering Committee on Quality Improvement and Management, Wolraich M, Brown L, Brown RT, DuPaul G, Earls M, Feldman HM, Ganiats TG, Kaplanek B, et al. ADHD: clinical practice guideline for the diagnosis, evaluation, and treatment of attention-deficit/hyperactivity disorder in children and adolescents. Pediatrics 2011; 128(5):1007-1022.

19. Schmitt J, Apfelbacher C, Chen C-M, Romanos M, Sausenthaler S, Koletzko S, Bauer C-P, Hoffmann U, Krämer U, Berdel D, et al. Infant-onset eczema in relation to mental health problems at age 10 years: results from a prospective birth cohort study (German Infant Nutrition Intervention plus). J Allergy Clin Immunol. 2010;125(2):404-10.

20. Schmitt J, Buske-Kirschbaum A, Roessner $V$. Is atopic disease a risk factor for attention-deficit/hyperactivity disorder? A systematic review. Allergy. 2010;65(12):1506-24.

21. Boris M, Goldblatt A. Pollen exposure as a cause for the deterioration of neurobehavioral function in children with autism and attention deficit hyperactive disorder: nasal pollen challenge. J Nutr Environ Med. 2004;14(1):47-54.

22. Biederman J, Milberger S, Faraone SV, Guite J, Warburton R. Associations between childhood asthma and ADHD: issues of psychiatric comorbidity and familiality. J Am Acad Child Adolesc Psychiatry. 1994:33(6):842-8.

23. Higgins J, Green S. Cochrane handbook for systematic reviews of interventions version 5.1. 0 [Updated 2011]. The Cochrane Collaboration: 2011. http://handbook.cochrane.org. Accessed 15 December 2015

24. Moher D, Liberati A, Tetzlaff J, Altman DG, The PRISMA Group. Preferred reporting items for systematic reviews and meta-analyses: the PRISMA statement. Ann Internal Med. 2009:151(4):264-9.

25. American Psychiatric Association. Diagnostic and statistical manual of mental disorders text-revision 4th ed. Washington: American Psychiatric Association Press; 2000.

26. National Institute for Health and Clinical Excellence. Attention deficit hyperactivity disorder: diagnosis and management. Clinical guideline[72]. London: NICE; 2016. https://www.nice.org.uk/guidance/cg72. Accessed 28 Mar 2016

27. Kim SY, Park JE, Lee YJ, Seo H-J, Sheen S-S, Hahn S, Jang B-H, Son H-J. Testing a tool for assessing the risk of bias for nonrandomized studies showed moderate reliability and promising validity. J Clin Epidemiol. 2013:66(4):408-14

28. Sterne JAC, Higgins JPT, Reeves BC, on behalf of the development group for ACROBAT-NRSI. A Cochrane risk of bias assessment tool: for nonrandomized studies of interventions (ACROBAT-NRSI) version 1.0.0; 2014. http://methods.cochrane.org/news/risk-bias-assessment-tool-nonrandomised-studies-interventions. Accessed 18 December 2015.

29. Review Manager (RevMan) [Computer program]. Version 5.3. Copenhagen: The Nordic Cochrane Centre, The Cochrane Collaboration; 2014

30. GRADEpro GDT. GRADEpro Guideline Development Tool [Software]. (Developed by Evidence Prime, Inc.): McMaster University; 2015. http://www. gradepro.org. Accessed 3 Feb 2016

31. Schünemann $H$, Brożek J, Guyatt $G H$, Oxman AD, editors. GRADE handbook for grading the quality of evidence and strength of recommendation using the GRADE approach [Updated October 2013]. The GRADE Working Group 2013. http://gdt.quidelinedevelopment.org/central_prod/_design/client/ handbook/handbook.html. Accessed 18 Dec 2015.

32. Guyatt GH, Oxman AD, Sultan S, Glasziou P, Akl EA, Alonso-Coello P, Atkins D, Kunz R, Brozek J, Montori V, et al. GRADE guidelines: 9. Rating up the quality of evidence. J Clin Epidemiol. 2011;64(12):1311-6.

33. Chen M-H, Su T-P, Chen Y-S, Hsu J-W, Huang K-L, Chang W-H, Bai Y-M. Attention deficit hyperactivity disorder, tic disorder, and allergy: is there a link? A nationwide population-based study. J Child Psychol Psychiatry. 2013; 54(5):545-51.

34. Hak E, de Vries TW, Hoekstra PJ, Jick SS. Association of childhood attentiondeficit/hyperactivity disorder with atopic diseases and skin infections? A matched case-control study using the General Practice Research Database. Ann Allergy Asthma Immunol. 2013;111(2):102-6.

35. Suwan $P$, Akaramethathip $D$, Noipayak P. Association between allergic sensitization and attention deficit hyperactivity disorder (ADHD). Asian Pac J Allergy Immunol. 2011;29(1):57-65.

36. Kwon HJ, Lee MY, Ha M, Yoo SJ, Paik KC, Lim J-H, Sakong J, Lee C-G, Kang D-M, Hong SJ, et al. The associations between ADHD and asthma in Korean children. BMC Psychiatry. 2014;14:70.

37. Romanos M, Gerlach M, Warnke A, Schmitt J. Association of attentiondeficit/hyperactivity disorder and atopic eczema modified by sleep disturbance in a large population-based sample. J Epidemiol Community Health. 2010;64(3):269-73.

38. Mill J, Curran S, Richards S, Taylor E, Asherson P. Polymorphisms in the dopamine D5 receptor (DRD5) gene and ADHD. Am J Med Genet B Neuropsychiatr Genet. 2004;125(1):38-42.

39. Ostadali MR, Ahangari G, Eslami MB, Razavi A, Zarrindast MR, Ahmadkhaniha HR, Boulhari J. The detection of dopamine gene receptors (DRD1-DRD5) 
expression on human peripheral blood lymphocytes by real time PCR. Iran J of Allergy Asthma Immunol. 2004;3(4):169-74.

40. Wu J, Xiao H, Sun H, Zou L, Zhu L-Q. Role of dopamine receptors in ADHD: a systematic meta-analysis. Mol Neurobiol. 2012;45(3):605-20.

41. Nakagome K, Imamura M, Okada H, Kawahata K, Inoue T, Hashimoto K, Harada H, Higashi T, Takagi R, Nakano K, et al. Dopamine D1-like receptor antagonist attenuates Th17-mediated immune response and ovalbumin antigen-induced neutrophilic airway inflammation. J Immunol. 2011;186(10):5975-82.

42. Bielory L. Allergic conjunctivitis and the impact of allergic rhinitis. Curr Allergy Asthma Rep. 2010;10(2):122-34.

43. Morar N, Willis-Owen SA, Moffatt MF, Cookson WO. The genetics of atopic dermatitis. J Allergy Clin Immunol. 2006;1 18(3):559.

44. Palmer CN, Irvine AD, Terron-Kwiatkowski A, Zhao Y, Liao H, Lee SP, Goudie DR, Sandilands A, Campbell LE, Smith FJ, et al. Common loss-of-function variants of the epidermal barrier protein filaggrin are a major predisposing factor for atopic dermatitis. Nat Genet. 2006;38(4):441-6.

45. Johnston LK, Chien KB, Bryce PJ. The Immunology of Food Allergy. J Immunol. 2014;192(6):2529-34.

46. Gratten J, Wray NR, Keller MC, Visscher PM. Large-scale genomics unveils the genetic architecture of psychiatric disorders. Nat Neurosci. 2014;17(6):782-90.

Submit your next manuscript to BioMed Central and we will help you at every step:

- We accept pre-submission inquiries

- Our selector tool helps you to find the most relevant journal

- We provide round the clock customer support

- Convenient online submission

- Thorough peer review

- Inclusion in PubMed and all major indexing services

- Maximum visibility for your research

Submit your manuscript at www.biomedcentral.com/submit
C Biomed Central 